\title{
A polarized view of string topology
}

\author{
Ralph L. Cohen * and Véronique Godin \\ Stanford University
}

November 13, 2018

Dedicated to Graeme Segal on the occasion of his 60th birthday.

\begin{abstract}
Let $M$ be a closed, connected manifold, and $L M$ its loop space. In this paper we describe closed string topology operations in $h_{*}(L M)$, where $h_{*}$ is a generalized homology theory that supports an orientation of $M$. We will show that these operations give $h_{*}(L M)$ the structure of a unital, commutative Frobenius algebra without a counit. Equivalently they describe a positive boundary, two dimensional topological quantum field theory associated to $h_{*}(L M)$. This implies that there are operations corresponding to any surface with $p$ incoming and $q$ outgoing boundary components, so long as $q \geq 1$. The absence of a counit follows from the nonexistence of an operation associated to the disk, $D^{2}$, viewed as a cobordism from the circle to the empty set. We will study homological obstructions to constructing such an operation, and show that in order for such an operation to exist, one must take $h_{*}(L M)$ to be an appropriate homological proobject associated to the loop space. Motivated by this, we introduce a prospectrum associated to $L M$ when $M$ has an almost complex structure. Given such a manifold its loop space has a canonical polarization of its tangent bundle, which is the fundamental feature needed to define this prospectrum. We refer to this as the "polarized Atiyah - dual" of $L M$. An appropriate homology theory applied to this prospectrum would be a candidate for a theory that supports string topology operations associated to any surface, including closed surfaces.
\end{abstract}

\section{Introduction}

Let $M^{n}$ be a closed, oriented manifold of dimension $n$, and let $L M$ be its free loop space. The "string topology" theory of Chas-Sullivan [3] describes a rich structure in the homology and equivariant homology of $L M$. The most basic operation is an intersection - type product,

$$
\circ: H_{q}(L M) \times H_{r}(L M) \longrightarrow H_{q+r-n}(L M)
$$

that is compatible with both the intersection product in the homology of the manifold, and the Pontrjagin product in the homology of the based loop space, $H_{*}(\Omega M)$. Moreover this product

${ }^{*}$ The first author was partially supported by a grant from the NSF 
structure extends to a Batalin-Vilkovisky algebra structure on $H_{*}(L M)$, and an induced Lie algebra structure on the equivariant homology, $H_{*}^{S^{1}}(L M)$. More recently Chas and Sullivan 4 described a Lie bialgebra structure on the rational reduced equivariant homology, $H_{*}^{S^{1}}(L M, M ; \mathbb{Q})$, where $M$ is embedded as the constant loops in $L M$.

These string topology operations and their generalizations are parameterized by combinatorial data related to fat graphs used in studying Riemann surfaces [3], [4], 17, [18, [5]. The associated field theory aspects of string topology is a subject that is still very much under investigation. In this paper we contribute to this investigation in the following two ways.

Recall that a two dimensional topological quantum field theory associates to an oriented compact one manifold $S$ a vector space $A_{S}$, and to any oriented cobordism $\Sigma$ between $S_{1}$ and $S_{2}$ a linear map $\mu_{\Sigma}: A_{S_{1}} \rightarrow A_{S_{2}}$. Such an assignment is required to satisfy various well known axioms, including a gluing axiom, and the requirement that the vector space associated to the empty one dimensional manifold, is the ground field $A_{\emptyset}=k$. Thus a TQFT assigns to a closed two dimensional manifold $\Sigma$, viewed as a cobordism from the empty set to itself, an invariant $\mu_{\Sigma} \in k$ which can be computed by viewing a closed manifold as a compositions of cobordisms between one manifolds.

Recall also that if $A=A_{S^{1}}$, then such a TQFT structure is equivalent to a Frobenius algebra structure on $A$ [15], 9], 1]. This is a unital, commutative algebra structure, $\mu: A \otimes A \rightarrow A$, together with a counit (or trace map ) $\theta: A \rightarrow k$ so that the composition $\theta \circ \mu: A \otimes A \rightarrow A \rightarrow k$ is a nondegenerate form. From the TQFT point of view, the unit in the algebra, $u: k \rightarrow A$ is the operation corresponding to a disk $D^{2}$ viewed as a cobordism from the empty set to the circle, and the counit $\theta: A \rightarrow k$ is the operation corresponding to the disk viewed as a cobordism from the circle to the empty set. The composition, $\theta \circ u: k \rightarrow A \rightarrow k$ is therefore the invariant corresponding to the sphere $S^{2}, \mu_{S^{2}} \in k$. Without the counit $\theta$ a Frobenius algebra is equivalent to a unital, commutative algebra $A$, together with a cocommutative coalgebra structure, $\Delta: A \rightarrow A \otimes A$ without counit, where $\Delta$ is a map of $A$-modules. From the TQFT point of view, a noncounital Frobenius algebra corresponds to a "positive boundary" TQFT, in the sense that operations $\mu_{\Sigma}$ are defined only when then the surface $\Sigma$ has a positive number of outgoing boundary components.

Let $h^{*}$ be a multiplicative generalized cohomology theory whose coefficient ring, $h^{*}$ (point) is a graded field (that is, every nonzero homogeneous element is invertible). Let $h_{*}$ be its associated generalized homology theory. Let $M^{n}$ be a closed, $n$ - dimensional manifold which is oriented with respect to this theory. Our first result, which builds on work of Sullivan [17, is that string topology operations can be defined to give a two dimensional positive boundary TQFT, with $A_{S^{1}}=h_{*}(L M)$. Said another way, we will prove the following.

Theorem 1. The homology of the free loop space $h_{*}(L M)$ has the structure of a Frobenius algebra without counit. The ground field of this algebra structure is the coefficient field, $h_{*}=h_{*}$ (point).

The construction of the TQFT operations corresponding to a surface $\Sigma$ will involve studying spaces of maps from a fat graph $\Gamma_{\Sigma}$ associated to the surface to $M$, and viewing that space as a finite codimension submanifold of a $(L M)^{p}$, where $p$ is the number of incoming boundary components 
of $\Sigma$. We will show that this allows the construction of a Thom collapse map for this embedding, which will in turn define a push-forward map $\iota !: h_{*}(L M)^{\otimes p} \rightarrow h_{*}\left(\operatorname{Map}\left(\Gamma_{\Sigma} ; M\right)\right.$. The operation $\mu_{\Sigma}$ will then be defined as the composition $\rho_{\text {out }} \circ \iota !$ where $\rho_{\text {out }}$ is induced by restricting a map from $\Gamma_{\Sigma}$ to $M$ to its outgoing boundary components.

The second goal of this paper is to investigate the obstructions to constructing a homological theory applied to the loop space which supports the string topology operations, and permits the definition of a counit in the Frobenius algebra structure, or equivalently, would eliminate the "positive boundary" requirement in the TQFT structure. Let $h_{*}^{\text {mid }}(L M)$ be such a conjectural theory. In some sense this would represent a "middle dimensional", or "semi-infinite" homology theory associated to the loop space, because of the existence of a nonsingular form $h_{*}^{\text {mid }}(L M) \times h_{*}^{\text {mid }}(L M) \rightarrow k$ analogous to the interesection form on the middle dimensional homology of an even dimensional oriented manifold.

We will see that defining a counit would involve the construction of a push-forward map for the embedding of constant loops $M \hookrightarrow L M$. Unlike the embeddings described above, this has infinite codimension. We will argue that this infinite dimensionality will force the use of an inverse limit of homology theories, or a "pro-homology theory" associated to the loop space. Using previous work of the first author and Stacey [8], we will show that there are obstructions to the construction of such a pro-object unless $M$ has an almost complex structure. In this case the tangent bundle of the loop space has a canonical complex polarization, and we will use it to define the "polarized loop space", $L M_{ \pm}$. This space fibers over $L M$, where the fiber over $\gamma \in L M$ is a representative of the polarization of the tangent space $T_{\gamma} L M$. We will examine various properties of $L M_{ \pm}$, including its equivariant properties. We will show that the pullback of the tangent bundle $T L M$ over $L M_{ \pm}$has a filtration that will allow us to define a prospectrum $L M_{ \pm}^{-T L M}$, which we call the (polarized) "Atiyah dual" of $L M$. We will end by describing how the application of an appropriate equivarant homology theory to this prospectrum should be a good candidate for studying further field theory properties of string topology. This will be the topic of future work, which will be joint with J. Morava and G. Segal.

The paper is organized as follows. In section 1 we will describe the type of fat graphs needed to define the string topology operations. These are chord diagrams of the sort introduced by Sullivan [17. We will define the topology of these chord diagrams using categorical ideas of Igusa [11 [12. Our main technical result, which we will need to prove the invariance of the operations, is that the space of chord diagrams representing surfaces of a particular diffeomorphism type is connected. In section 2 we use the space of chord diagrams to define the string topology operations and prove theorem 1. The operations will be defined using a homotopy theoretic construction (the "Thom collapse map") generalizing what was done by the first author and Jones in [5]. In section 3 we discuss the issues surrounding the unit and the counit, and describe the obstructions to the existence of a counit or trace in the Frobenius algebra structure. Motivated by these observations, in section 4 we describe the "polarized Atiyah dual" of the loop space of an almost complex manifold, and study 
its properties.

The authors are grateful to J. Morava, G. Segal, and D. Sullivan for many inspiring conversations about the topics of this paper.

\section{Fat graphs and Sullivan chord diagrams}

Recall from [13 [16 that a fat graph is a graph whose vertices are at least trivalent, and where the edges coming into each vertex come equipped with a cyclic ordering. Spaces of fat graphs have been used by many different authors as an extremely effective tool in studying the topology and geometry of moduli spaces of Riemann surfaces. The essential feature of a fat graph is that when it is thickened, it produces a surface with boundary, which is well defined up to homeomorphism.

The approach we will use to the topology of the space of fat graphs follows that of Igusa [11] 12. We begin by recalling some of his constructions.

In 11] (chapter 8), the notion of metric fat graph was defined as follows.

Definition 1. A (topological) metric fat graph is a fat graph that has the structure of a compact metric space $\Gamma$ satisfying the following properties:

1. Let $\Gamma_{0}$ be $\Gamma$ with the vertices removed. Then $\Gamma_{0}$ is locally isometrically equivalent to $\mathbb{R}$.

2. If $x, y \in \Gamma$ lie in the same path component then each minimal path from $x$ to $y$ in $\Gamma$ is isometrically equivalent to a closed interval of length $d=d(x, y)$.

3. $d(x, y)=1$ if $x, y$ lie in different path components of $\Gamma$.

4. Each component of $\Gamma$ has total length equal to 1.

Let $\mathcal{F}_{n}(g)$ be the set of metric fat graphs representing surfaces of genus $g$ with $n$ punctures. Igusa topologized this space as the geometric realization of a simplicial set which he showed was homotopy equivalent to the nerve of the category $\mathcal{F} a t_{n}(g)$ defined as follows.

The objects of $\mathcal{F} a t_{n}(g)$ are fat graphs (with no lengths assigned to the edges), and the morphisms are maps of fat graphs $f: \Gamma_{1} \rightarrow \Gamma_{2}$ (i.e maps of the underlying simplicial complexes that preserve the cyclic orderings) satisfying the following properties:

1. The inverse image of any vertex is a tree.

2. The inverse image of an open edge is an open edge.

The main result about these spaces is that $\mathcal{F}_{n}(g) \simeq\left|\mathcal{F}_{a} t_{n}(g)\right|$ is homotopy equivalent to the classifying space, $B \mathcal{M}_{g, n}$, where $\mathcal{M}_{g, n}$ is the mapping class group of genus $g$ surfaces with $n$ marked points.

Given a metric fat graph $\Gamma$, one has "boundary cycles" defined in the following way. Pick an edge and choose an orientation on it. Traversing that edge in the direction of the its orientation 
leads one to a vertex. Proceed with the next edge emanating from that vertex in the cyclic ordering, and give it the orientation pointing away from that vertex. Continuing in this way, one traverses several edges, eventually returning to the original edge, with the original orientation. This yields a "cycle" in the set of oriented edges. This represents a boundary component of a given length (the sum of the lengths of the edges in the cycle). One can partition the set of all oriented edges into a set of cycles, which enumerates the boundary components of the surface represented by $\Gamma$. Notice that the cycle structure of the oriented edges completely determines the combinatorial data of the fat graph.

As mentioned, each boundary cycle in a metric fat graph has an orientation and a metric on it, giving it a total length. If in addition, we introduce a marked point for each boundary cycle, this would yield a parameterization of each boundary component. (This parameterization is an orientation preserving local diffeomorphism from the unit circle $S^{1}$ to the boundary cycle obtained in the following way. First map the unit circle to the concentric circle of length $\ell$, the total length of the boundary cycle, by radial expansion. Then map that circle to the boundary cycle in an orientation and length preserving way, starting by mapping the basepoint of the circle to the marked point of the boundary cycle.) Notice that it is possible that two marked points lie on the same edge, and indeed a single point on an edge might have a "double marking". This is because a single edge with its two orientations might lie in two different boundary cycles.

We call the space of metric fat graphs representing surfaces of genus $g$ with $n$ boundary components, that come equipped with marked points on the the boundary cycles, $\mathcal{F}_{n}^{\mu}(g)$. This is the space of marked metric fat graphs. Using Igusa's simplicial set construction, one sees that $\mathcal{F}_{n}^{\mu}(g)$ can be topologized so that the projection map that forgets the markings,

$$
p: \mathcal{F}_{n}^{\mu}(g) \rightarrow \mathcal{F}_{n}(g)
$$

is a quasifibration whose fiber is the space of markings on a fixed fat graph, which is homeomorphic to the torus $\left(S^{1}\right)^{n}$. The topology of the space of marked metric fat graphs is studied in detail in [10] with applications to specific combinatorial calculations.

For the purposes of constructing the string topology operations, we will use a particular type of fat graph due to Sullivan.

Definition 2. A "Sullivan chord diagram" of type $(g ; p, q)$ is a fat graph representing a surface of genus $g$ with $p+q$ boundary components, that consists of a disjoint union of $p$ disjoint closed circles together with the disjoint union of connected trees whose endpoints lie on the circles. The cyclic orderings of the edges at the vertices must be such that each of the $p$ disjoint circles is a boundary cycle. These $p$ circles are referred to as the incoming boundary cycles, and the other $q$ boundary cycles are referred to as the outgoing boundary cycles.

The ordering in the diagrams that follow are indicated by the clockwise cyclic ordering of the plane. 


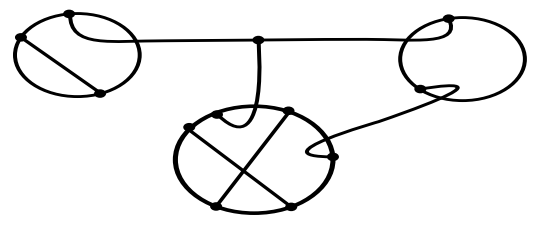

Figure 1: Sullivan chord diagram

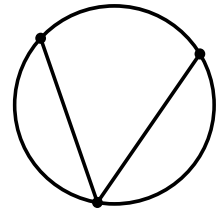

(a) c

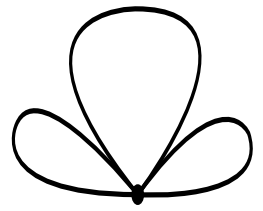

(b) $\mathrm{S}(\mathrm{c})$

Figure 2: Collapsing of ghost edges

In a Sullivan chord diagram, the vertices and edges that lie on one of the $p$ disjoint circles will be referred to as circular vertices and circular edges respectively. The others will be referred to as ghost vertices and edges.

To define the topology on the space of metric chord diagrams, we first need to define the space of metric fat graphs with distinguished incoming and outgoing boundary components. Namely, let $\mathcal{F}_{p, q}(g)$ be the space of metric fat graphs representing surfaces of genus $g$ with $p+q$ boundary components, where $p$ of the boundary components are distinguished as "incoming". The remaining boundary components are "outgoing". Igusa's simplicial construction of $\mathcal{F}_{n}(g)$ defines a model of $\mathcal{F}_{p, q}(g)$ as the geometric realization of a simplicial set. Moreover this space is homotopy equivalent to the realization of the nerve of the category $\mathcal{F} a t_{p, q}(g)$ defined as above, with the additional feature that the objects have $p$ distinguished incoming cycles, and the morphisms preserve this structure.

Consider the space of "metric chord diagrams", $\mathcal{C F}_{p, q}(g)$ defined to be the subspace of the metric fat graphs $\mathcal{F}_{p, q}(g)$ whose underlying graphs are Sullivan chord diagrams of type $(g ; p, q)$. By the definition of the simplicial structure of $\mathcal{F}_{p, q}(g)$, one sees that $\mathcal{C F}_{p, q}(g)$ is actually the realization of a simplicial subset. Moreover, if $\mathcal{C} \mathcal{F} a t_{p, q}(g)$ is the full subcategory of $\mathcal{F} a t_{p, q}(g)$ whose objects are chord diagrams of type $(g ; p, q)$, then Igusa's argument shows that the space of metric chord diagrams $\mathcal{C F}_{p, q}(g)$ is homotopy equivalent to the realization of the nerve of the category $\mathcal{C} \mathcal{F} a t_{p, q}(g)$.

Given a metric chord diagram $c \in \mathcal{C F}_{p, q}(g)$, there is an associated metric fat graph, $S(c)$, obtained from $c$ by collapsing each ghost edge to a vertex. There is an induced cyclic ordering on the vertices of $S(c)$ so that the collapse map $\pi: c \rightarrow S(c)$ is a map of fat graphs in $\mathcal{F}_{p, q}(g)$. Figure 2 describes this collapse map. 
We will define a marking of a Sullivan chord diagram $c$ to be a marking of the boundary cycles of the associated fat graph $S(c)$. Notice that on the incoming boundary components, this is equivalent to choosing a marked point on each of the circles making up the chord diagram. For the outgoing boundary cycles, this is equivalent to choosing a marked point on a circular edge in the boundary cycles subject to the identification that if two circular vertices in the same outgoing cycle are connected by a sequence of ghost edges, then we identify markings at two such vertices. Observe that since the ghost edges form a disjoint union of contractible trees, every outgoing boundary cycle must contain at least one circular edge.

We let $\mathcal{C F}_{p, q}^{\mu}(g)$ denote the space of all marked metric chord diagrams. Like with the full space of marked fat graphs, this space can be topologized in a natural way so that the projection map that forgets the markings,

$$
p: \mathcal{C F}_{p, q}^{\mu}(g) \longrightarrow \mathcal{C F}_{p, q}(g)
$$

is a quasifibration, with fiber over a metric chord diagram $c$ the the space of markings of $S(c)$, which is equivalent to the torus $\left(S^{1}\right)^{p+q}$. Again, the topology of these spaces of marked chord diagrams will be studied in detail in [10].

The space of marked, metric chord diagrams $\mathcal{C F}_{p, q}^{\mu}(g)$ will be used in the next section to parameterize the string topology operations. In order to prove the appropriate invariance property of these operations necessary to prove theorem 1] we will need the following result.

Theorem 2. The space $\mathcal{C F}_{p, q}^{\mu}(g)$ is path connected.

Proof. Because of quasifibration (2), it suffices to prove that the space of unmarked metric chord diagrams $\mathcal{C F}_{p, q}(g)$ is connected. However as remarked earlier, this space is homotopy equivalent to the nerve of the category $\mathcal{C F} a t_{p, q}(g)$.

Now a morphism between objects $a$ and $b$ in a category determines a path from $a$ to $b$ in the geometric realization of its nerve. By reversing orientation, a morphism from $b$ to $a$ also determines a path from $a$ to $b$ in the geometric realization. We refer to this as an inverse morphism from $a$ to $b$. (Formally it is a morphism from $a$ to $b$ in the opposite category.) Therefore to prove this theorem it suffices to build, for any chord diagram in our space, a sequence of morphisms and inverses from it to a fixed chord diagram $\Gamma_{0}$. Since a morphism in $\mathcal{C} \mathcal{F} a t_{p, q}(g)$ collapses trees to vertices, we refer to such morphisms as "collapses". We refer to an inverse morphism as an "expansion". In the

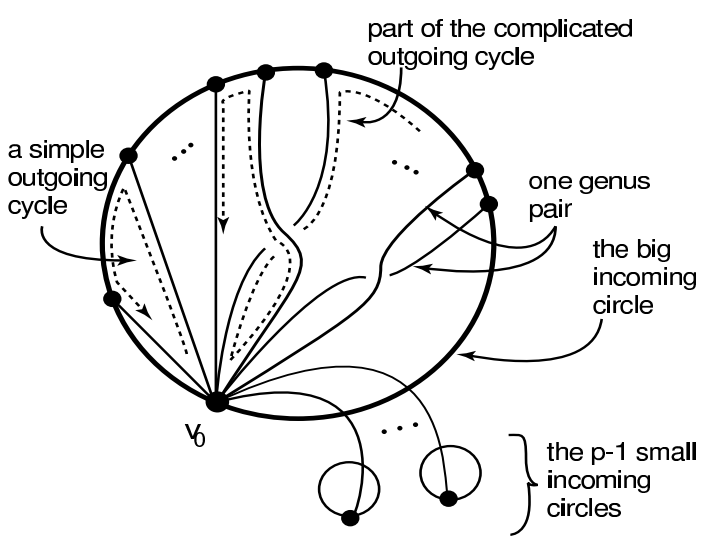

Figure $3: \Gamma_{0}$ following diagrams, dashed lines will represent boundary cycles. 
We will choose our basepoint $\Gamma_{0}$ as in figure 3 In $\Gamma_{0}, p-1$ of the incoming circles contain only one vertex. There is also a distinguished vertex $v_{0}$ in the $p^{\text {th }}$ incoming circle (which we refer to as the "big circle"). Moreover $q-1$ outgoing components share the same structure : they can be traced by going from $v_{0}$ along a chord edge whose other vertex also lies on the big circle, going along the next circle edge in the cyclic ordering, and then going along the next chord edge back to $v_{0}$. In the last outgoing boundary component positive genus is produced by pairs of chord edges twisted, as shown. (These pairs add two generators in the fundamental group of our surface but do not affect the number of boundary components, therefore they create genus.) Notice also that except for $v_{0}$ all of the vertices of $\Gamma_{0}$ are trivalent. So, in $\Gamma_{0}$ the complexity is concentrated in the big incoming circle, the last outgoing boundary circle, and one vertex $v_{0}$.

To prove our claim we'll start from any chord diagram (object) in $\mathcal{C} \mathcal{F} a t_{p, q}(g)$ and get to $\Gamma_{0}$ by a sequence of collapses and expansions. In our diagrams, the collapses will be denoted by an arrow going right and the expansions by an arrow going left. (The arrow hence follows the direction of the corresponding morphism in our category $\mathcal{C F} a t_{p, q}(g)$.) Since, in a Sullivan chord diagram, the incoming boundaries are represented by disjoint circles, a chord edge between two circular vertices cannot be collapsed. Remember also that the ghost edges need to form a disjoint union of trees. Hence if both vertices of a circular edge are part of the same tree of ghost edges (same connected component of the ghost structure), this circular edge cannot be collapsed. We will call an edge "essential" if it cannot be collapsed. That is, it is either a circular edge and its collapse would create a non-trivial cycle in the ghost structure, or it is a chord edge between two circular vertices.

Throughout this proof, letters from the beginning of the alphabet will be used to label edges that are on the verge of being collapsed, and letters from the end of the alphabet will be used to label edges that have just been created, via an expansion. We will start by assuming that all nonessential edges have been collapsed. We are now ready to build our path.

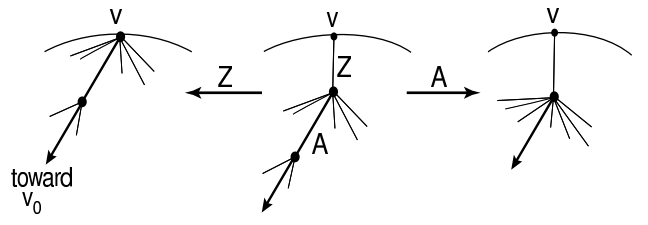

Figure 4: Pushing edges

The first step will be to find a path to a chord diagram with a distinguished vertex $v_{0}$, the only one with more than three edges emanating from it. Choose $v_{0}$ to be any vertex. For any vertex $v$, other than $v_{0}$, having more than three edges, we will "push" the edges of $v$ toward $v_{o}$ by a sequence of expansions and collapses. This is done as follows.

Since all edges are essential, the vertices of any circular edge are part of the same connected componnent of the ghost structure. This means that for any circular edge with vertices $a$ and $b$, there exists a path of ghost edges connecting $a$ and $b$. We can therefore choose a path $\gamma$ from $v$ to $v_{0}$ contained completely in the the ghost structure. Following figure 4 we can push the edges of $v$ a step closer to $v_{0}$. (Notice that $A$ isn't essential since it ends at a newly-created ghost vertex.) Repeating this process allows us to complete this step.

We now have a distinguished vertex $v_{0}$ the only one with more than one ghost edge. Note that 


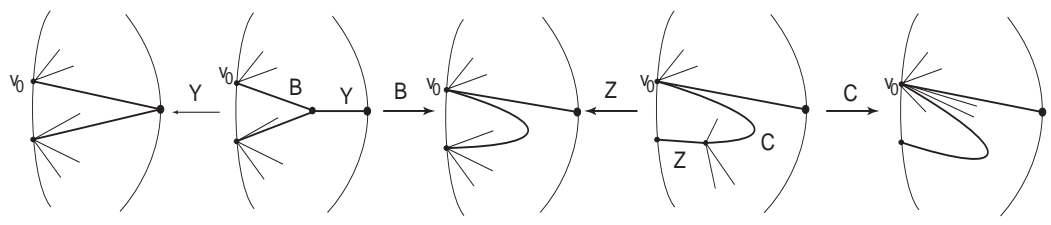

Figure 6: Pushing edges towards $v_{0}$

there is a unique ordering of the edges emanating from $v_{0}$ that is compatible with the cyclic order, and so that one of the circular edges of $v_{0}$ is first in the order, and the other circular edge is last in the order. We will think of this ordering of edges as passing from left to right.

We will next simplify the incoming circles. In this step all of the incoming cycles but the first will be brought down to one edge. To achieve this we first need to notice that all ghost edges have $v_{0}$ as one of their vertices. To see this, notice that a ghost edge between two other vertices would be in itself a connected component of the ghost structure. We could therefore collapse one of the circular edges adjacent to

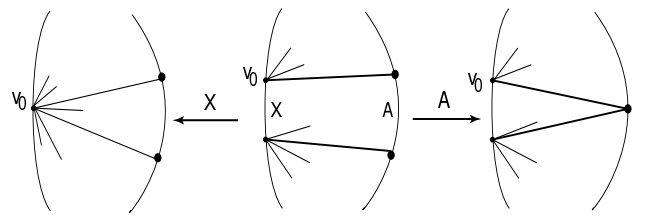

Figure 5: Getting rid of one edge it (in the cyclic ordering), and join two connected components of the ghost structure. But since we only have essential edges in our graph, we know that this isn't possible. Therefore all ghost edges have $v_{0}$ as a vertex.

Now take any incoming boundary circle containing more than one edge (other than the big circle). We cannot simply collapse one of its edges as it would create a cycle in the ghost structure (the union of the ghost edges) through $v_{0}$. But as seen in figure 5 the addition of an edge $X$ (by an expansion) close to $v_{0}$, renders $A$ nonessential and it can be collapsed.

After this has been done, there will be two vertices other than $v_{0}$ that have more than three edges. As shown in the figure 6, this number can be brought back down by a simple play with chord edges. Observe that in this procedure there is no risk of collapsing an essential edge.

Repeating this process we reduce the number of vertices on these incoming circles down to one per circle. Notice that our chord diagram still has a unique non-trivalent vertex $v_{0}$ and no nonessential edges.

Now the $p-1$ small incoming circles have a unique vertex with a unique ghost edge linking it with $v_{0}$ on the big incoming circle. Using their simple structure we will be able to change the order of their ghost edges coming into $v_{0}$ and any other ghost edge also coming into $v_{0}$, by the procedure shown in the seven pictures of figure 7 


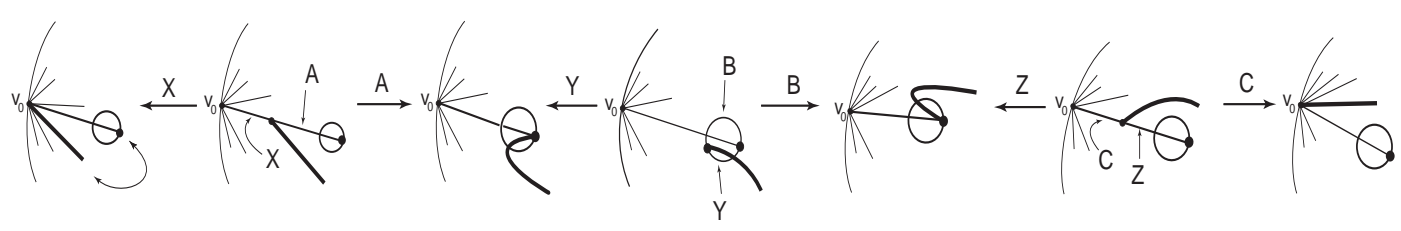

Figure 7: Switching an incoming circle and an edge

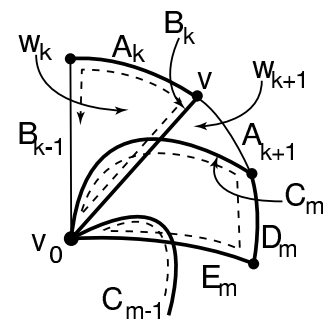

Figure 8:

Our next step is to simplify the structure of $q-1$ outgoing boundary components (all but the last one). This will be achieved by lowering the number of edges involved in the tracing of these boundary components to a minimum (down to three edges except for the first boundary which only needs two). We will use the term clean to refer to this simple form. Notice that this is exactly the situation in $\Gamma_{0}$.

Note also that each of the outgoing boundaries has an edge on the big circle. (The cleaned boundaries will only have one such edge.) Assume by induction that $k-1$ outgoing boundaries have already been cleaned.

Assume also that these cleaned boundaries have been pushed to the left of the big incoming circle meaning that their (unique) incoming edge is situated to the left of all the incoming edges associated to the uncleaned boundaries (no uniqueness here) and that their ghost edges at $v_{0}$ are attached left of all other ghost edges. Assume also that at least two outgoing boundaries still need to be cleaned. Firstly we will clean one more outgoing boundary and secondly we will push it left.

Since we have moved all the clean boundaries to the left, we will have two successive circular edges, $A_{k}$ and $A_{k+1}$, on our big circle that are part of two different outgoing boundaries, $w_{k}$ and $w_{k+1}$, that are still unclean. Let $v$ be the common circular vertex of $A_{k}$ and $A_{k+1}$ and let $B_{k}$ be the ghost edge coming into $v . B_{k}$ can be thought of as separating these two boundaries. In particular the cycles representing both $w_{k}$ and $w_{k+1}$ include $B_{k}$ (with two different orientations). We will now push all the extra edges involved in the tracing of $w_{k}$ to the right of $B_{k}$ and hence into $w_{k+1}$. Using our previous work, we move all the small incoming circles out of our left boundary component $w_{k}$ and into $w_{k+1}$. After this all the ghost edges involved in the cycle representing $w_{k}$ start at $v_{0}$ and end on the big incoming circle. Using this, the cycle traced by $w_{k}$ is $\left(B_{k-1}, E_{1}, D_{1}, \ldots C_{m-1}, E_{m}\right.$, $D_{m}, C_{m}, B_{k}, A_{k}$ ) where the $E$ 's and the $C$ 's are ghost edges from $v_{0}$ to the big circle and the $D$ 's are circular edges on the big circle. Figure 8 shows the last part of this cycle of edges $\left(C_{m-1}, E_{m}\right.$, $\left.D_{m}, C_{m}, B_{k}, A_{k}, B_{k-1}\right)$. In figure 9 the start of $B_{k}$ is glided along the edges $C_{m} D_{m}$ and finally $E_{m}$. After these glidings $w_{k}$ will skip $E_{m}, D_{m}$ and $C_{m}$, and the tracing of $w_{k}$ will go directly from $C_{m-1}$ to $B_{k}$. $B_{k}$ is thickened on each of the pictures to help visualize the process. Notice that in the last image, $w_{k}$ is now simpler since it doesn't involve the edges $\left(E_{m}, D_{m}\right.$ and $\left.C_{m}\right)$. We can repeat this process until we only have $B_{k}, A_{k}$ and $B_{k-1}$ left in the tracing of the left boundary component.

To make sure we don't interfere with this boundary cycle when making subsequent rearrange- 


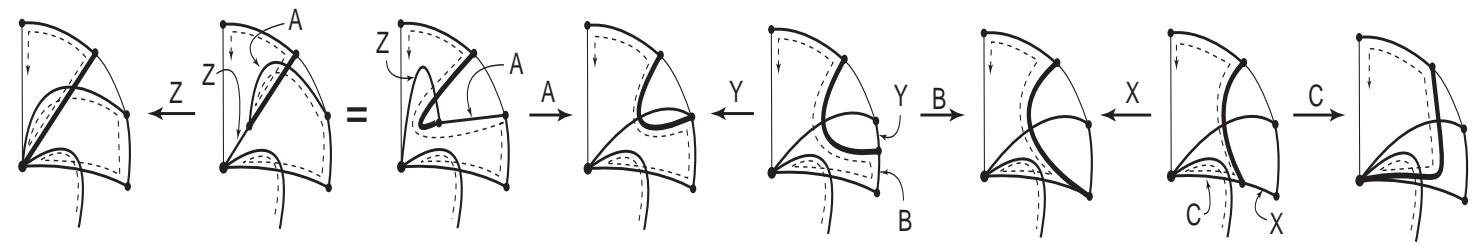

Figure 9: Skipping three edges

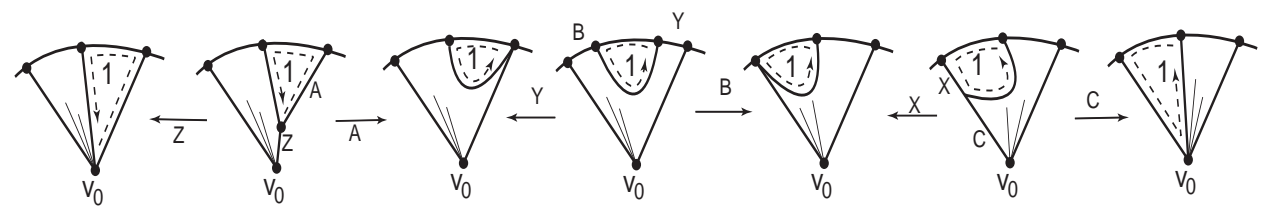

Figure 10: Moving boundary 1 to the left

ments, we will move it to the left of all the remaining uncleaned boundaries. Follow figure 10 to see how to switch this boundary (labelled 1) with the one directly on the left of it. After this, we'll have once again a chord diagram with the leftmost outgoing cycles simplified. Inductively we'll be able to clean all of them but the last one.

We are now very close to our goal. We have the

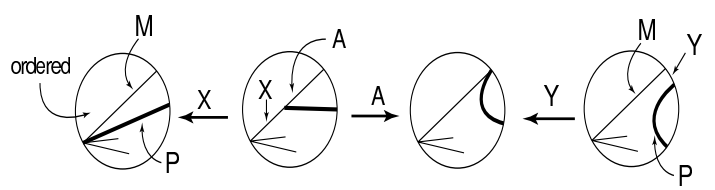

Figure 11: Moving $P$ away incoming and the outgoing boundaries in the right place and in the right form. The only issue now rests with the last outgoing boundary component. The one that now includes all the "genus creating" edges. To finally reach $\Gamma_{0}$, we need to untangle these into twisted (crossed) pairs. This is done by induction on the number of pairs of such edges left to untangle.

Choose $M$ to be first genus creating edge (in the ordering at $v_{0}$ ) that has not yet been paired in our inductive process. Our first step is to find an edge that "crosses it", meaning that it starts on the right of $M$ at $v_{0}$ and ends up on the left of $M$ on the big circle. Take $P$ to be the next edge at $v_{0}$. If $P$ ends up on the left of $M$, we have our edge and we are ready to apply the second step. If this is not the case, we'll move $P$ along $M$ as shown in figure 11 and restart this procedure but with $P$ out of the way. (We will therefore consider the next edge as a possible crossing for $M$.) Since both sides of $M$ are part of the same boundary component meaning that both orientations of $M$ appear in the boundary cycle (the last outgoing one), the tracing of this component moves from the edges on the right of $M$ to the edges on the left of $M$. This implies that there is at least one edge that starts on the right of $M$ at $v_{0}$ and ends on the left of $M$ on the big circle. So by moving through the edges $P$ we will find one of these crossing edges and this step will be completed. 


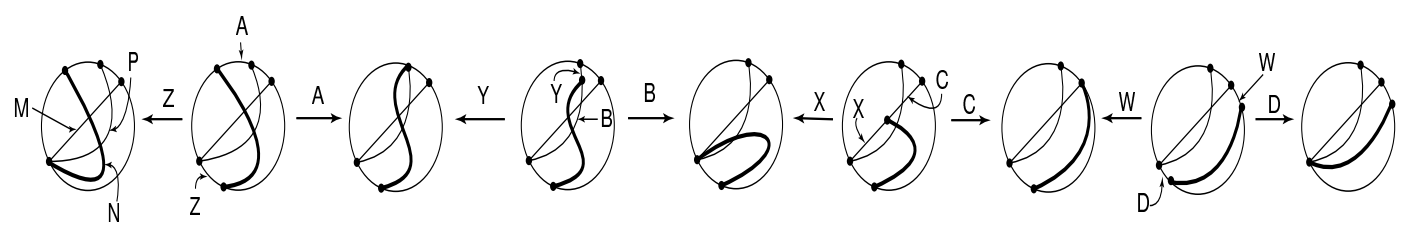

Figure 12: Separating the pair $M-P$ from $N$

Now we have our pair of edges $M$ and $P$. But we want more, we would like to have $M$ and $P$ completely separate from the other edges as in $\Gamma_{0}$. Hence any edge $N$ that is somehow intertwined with $M$ and $P$ needs to be moved. Since everything on the left of $M$ and $P$ at $v_{0}$ is already in proper order, this $N$ will always start on the right of $M$ and $P$ at $v_{o}$ (and end up on the big incoming circle). But there are still two ways that $N$ might be intertwined with our pair depending on exactly where it ends up : it could either end between $M$ and $P$ or on the left of these edges. Figure 12 shows how to glide the edge $N$ along first $M$ and then $P$ so that in the final image it ends up on the right of $M$ and $P$. A similar operation would get rid of the edges landing between $M$ and $P$.

Before restarting these steps for isolating the next pair, we need to bring a lot of edges back to $v_{0}$. For example in figure $11 P$ ends up completely disconnected from $v_{0}$ and from the rest of the ghost structure. To achieve this we will first collapse all nonessential edges and then we'll reduce the number of edges on the non- $v_{0}$ vertices down to three as done in figure 4 Note that $M$ and $P$ can be kept isolated while all of this is done.

After this process, all the genus edges are paired and twisted properly. We can finally put the ghost edges connecting the small circles to $v_{0}$ in their correct position. Our chord diagram has now one big incoming circle and a special vertex $v_{0}$ the only non-trivalent vertex. It has $p-1$ small "onevertexed" incoming circles linked with $v_{0}$ by one ghost edge landing in the last outgoing puncture. It has $q-1$ simple outgoing boundary components positioned on the left of the big incoming circle. The remaining outgoing component (situated on the extreme right of the big incoming circle) contains all the genus edges and the ghost edges linking the big incoming circles with the small ones. Moveover the genus edges are isolated into twisted pairs. This means that the cycles associated to the different boundary components are exactly the same in our chord diagram and in $\Gamma_{0}$. But we know that these cycles determine completely a fat graph. Hence we have succeeded. We have shown how to connect a random chord diagram to $\Gamma_{0}$ by a sequence of collapses and expansions.

We now know that the realization of the nerve of $\mathcal{C F} a t_{p, q}(g)$ is connected. But we have already argued that this implies that the space of marked metric chord diagrams $\mathcal{C F}_{p, q}^{\mu}(g)$ is connected. This completes the proof of the theorem. 


\section{The Thom collapse map and string topology operations}

In this section we use fat graphs to define the string operations, and will prove theorem 1 stated in the introduction.

As in the introduction, let $h^{*}$ be a multiplicative generalized cohomology theory. Such a theory is represented by a ring spectrum $E_{h}$. Assume furthermore that the coefficient ring , $h_{*}=h^{*}($ point $)=\pi_{*}\left(E_{h}\right)$ is a graded field (every nonzero homogeneous element is invertible). Recall that a $k$-dimensional vector bundle $\zeta \rightarrow M$ is oriented with respect to $h^{*}$, if there is a Thom isomorphism between the cohomology of the manifold, and that of the Thom space, $h^{*}(M) \cong h^{*+k}\left(M^{\zeta}\right)$. We say that a manifold $M$ is oriented with respect to $h^{*}$ if its tangent bundle $T M$ is oriented with respect to $h^{*}$. For the rest of this section we assume that $h^{*}$ is such a cohomology theory and $M$ is a compact, $n$-dimensional $h^{*}$-oriented manifold.

Let $L M$ denote the space of piecewise smooth maps, $\gamma: S^{1} \rightarrow M$. Let $\Sigma$ be an oriented surface of genus $g$ with $p+q$ boundary components, where $p$ of the components are viewed as incoming, and $q$ of the components are viewed as outgoing. We write $\partial_{i n} \Sigma$ and $\partial_{\text {out }} \Sigma$ for the incoming and outgoing components of the boundary $\partial \Sigma$. Assume one has a fixed parameterization of these boundary components, $\rho_{\text {in }}: \coprod_{p} S^{1} \rightarrow \partial_{\text {in }} \Sigma$ and $\rho_{\text {out }}: \coprod_{q} S^{1} \rightarrow \partial_{\text {out }} \Sigma$. Our goal in this section is to construct an operation

$$
\mu_{\Sigma}: h_{*}(L M)^{\otimes p} \longrightarrow h_{*}(L M)^{\otimes q}
$$

that will define a positive boundary TQFT. The operations in traditional homology theory are due to Sullivan [17]. In order to define these operations for generalized theories, we need to investigate the homotopy theory foundations of their constructions. The idea for doing this is the following.

Consider the space of smooth maps, $\operatorname{Map}(\Sigma, M)$. Using the above parameterizations, restriction to the incoming and outgoing boundary components defines a diagram

$$
(L M)^{q} \stackrel{\rho_{\text {out }}}{\longleftarrow} \operatorname{Map}(\Sigma, M) \stackrel{\rho_{\text {in }}}{\longrightarrow}(L M)^{p} .
$$

If $q>0$, we will define a "push-forward" map

$$
\left(\rho_{i n}\right) !: h_{*}\left((L M)^{p}\right) \rightarrow h_{*+\chi}(\Sigma) n(M a p(\Sigma, M))
$$

where $\chi(\Sigma)=2-2 g-p-q$ is the Euler characteristic of $\Sigma$. The operation $\mu_{\Sigma}$ will be defined to be the composition

$$
\mu_{\Sigma}=\left(\rho_{\text {out }}\right)_{*} \circ\left(\rho_{\text {in }}\right) !: h_{*}\left((L M)^{p}\right) \rightarrow h_{*+\chi(\Sigma) n}(\operatorname{Map}(\Sigma, M)) \rightarrow h_{*+\chi}(\Sigma) n\left((L M)^{q}\right) .
$$

So much of the work in proving theorem 1 is the definition of the push-forward map $\left(\rho_{i n}\right)$ !. For this we will make use of the space of marked, metric chord diagrams $\mathcal{C F}_{p, q}^{\mu}(g)$ studied in section one.

For $c \in \mathcal{C F}_{p, q}^{\mu}(g)$, let $\operatorname{Map}_{*}(c, M)$ be the space of continuous maps $f: c \rightarrow M$, smooth on each edge, which is constant on each ghost edge. Equivalently, this is the full space of maps $S(c) \rightarrow M$ where $S(c)$ is the marked metric fat graph described in the previous section (obtained from $S(c)$ by 
collapsing the ghost edges to points). Since each chord component is a tree and therefore contractible, this mapping space is homotopy equivalent to the space of all continuous maps, $c \rightarrow M$, which in turn is homotopy equivalent to the smooth mapping space, $\operatorname{Map}(\Sigma, M)$. Furthermore, the markings on $S(c)$ induce parameterizations of the incoming and outgoing boundary components of $c$ define a diagram modeling the homotopy type of diagram (3):

$$
(L M)^{q} \stackrel{\rho_{\text {out }}}{\longleftarrow} \operatorname{Map}_{*}(c, M) \stackrel{\rho_{\text {in }}}{\longrightarrow}(L M)^{p} .
$$

Since $\operatorname{Map}_{*}(c, M)$ is the same as the space of all continuous maps $\operatorname{Map}(S(c), M)$, that are smooth on each circle component, it is clear that the restriction to the incoming boundary components,

$$
\rho_{\text {in }}: \operatorname{Map}_{*}(c, M) \longrightarrow(L M)^{p}
$$

is an embedding of infinite dimensional manifolds, but it has finite codimension. We now consider its normal bundle.

Let $v(c)$ be the collection of circular vertices of a chord diagram $c$. Let $\sigma(c)$ be the collection of vertices of the associated graph $S(c)$. The projection map $\pi: c \rightarrow S(c)$ determines a surjective set map, $\pi_{*}: v(c) \rightarrow \sigma(c)$. For a vertex $v \in \sigma(c)$, we define the multiplicity, $\mu(v)$, to be the cardinality of the preimage, $\# \pi^{-1}(v)$. Let $M^{\sigma(c)}$ and $M^{v(c)}$ be the induced mapping spaces from these vertex collections. Then $\pi$ induces a diagonal map

$$
\Delta_{c}: M^{\sigma(c)} \longrightarrow M^{v(c)}
$$

The normal bundle of this diagonal embedding is the product bundle,

$$
\nu\left(\Delta_{c}\right)=\prod_{v \in \sigma(c)}(\mu(v)-1) T M \rightarrow \prod_{v \in \sigma(c)} M=M^{\sigma(c)} .
$$

Here $k \cdot T M$ is the $k$-fold Whitney sum of the tangent bundle. Since $\sum_{v \in \sigma(c)} \mu(v)=v(c)$, the fiber dimension of this bundle is $(v(c)-\sigma(c)) n$. An easy exercise verifies that $(v(c)-\sigma(c))=-\chi\left(\Sigma_{c}\right)$, minus the Euler characteristic of a surface represented by $c$.

Now remember that the markings of the the incoming boundary components of $S(c)$ define parameterizations of the incoming boundary components of $c$, since these cycles only consist of circular edges. Now using these parameterizations we can identify $(L M)^{p}$ with $M a p\left(c_{1} \sqcup \cdots \sqcup c_{p}, M\right)$. Consider the evaluation map $e_{c}:(L M)^{p} \rightarrow M^{v(c)}$ defined on an element of $\gamma \in(L M)^{p}$ by evaluating $\gamma$ on the circular vertices. Similarly, define

$$
e_{c}: \operatorname{Map}_{*}(c, M) \rightarrow M^{\sigma(c)}
$$

by evaluating a map $f: S(c) \rightarrow M$ on the vertices. These evaluation maps are fibrations, and notice 
that the following is a pull-back square:

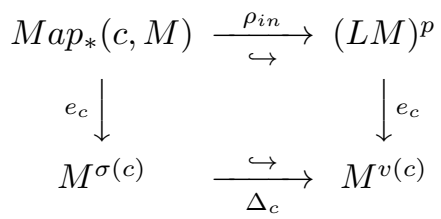

By taking the inverse image of a tubular neighborhood of the embedding $\Delta_{c}$, one has the following consequence.

Lemma 3. $\rho_{\text {in }}: \operatorname{Map}_{*}(c, M) \hookrightarrow(L M)^{p}$ is a codimension $-\chi\left(\Sigma_{c}\right) n$ embedding, and has an open neighborhood $\nu(c)$ diffeomorphic to the total space of the pullback bundle, $e_{c}^{*}\left(\nu\left(\Delta_{c}\right)\right)=e_{c}^{*}\left(\prod_{v \in \sigma(c)}(\mu(v)-\right.$ 1)TM). The fiber of this bundle over a map $f: c \rightarrow M$ is therefore given by

$$
\nu(c)_{f}=\bigoplus_{v \in \sigma(c)} \bigoplus_{(\mu(v)-1)} T_{f(v)} M
$$

Let $\operatorname{Map}_{*}(c, M)^{\nu(c)}$ be the Thom space of this normal bundle. This result allows us to define a Thom collapse map $\tau:(L M)^{p} \rightarrow \operatorname{Map}_{*}(c, M)^{\nu(c)}$ defined, as usual, to be the identity inside the tubular neighborhood, and the basepoint outside the tubular neighborhood.

Now let $h^{*}$ be a generalized cohomology theory as before. By the above description of the bundle $\nu(c)$ we see that since $M$ is $h^{*}$-oriented, the bundle $\nu(c)$ is $h^{*}$-oriented. This defines a Thom isomorphism,

$$
t: h_{*}\left(\operatorname{Map}_{*}(c, M)^{\nu(c)}\right) \cong h_{*+\chi\left(\Sigma_{c}\right) n}\left(\operatorname{Map}_{*}(c, M)\right) .
$$

Now since we are assuming that the coefficient ring $h_{*}=h_{*}$ (point) is a graded field, the Kunneth spectral sequence collapses, and hence

$$
h_{*}(X \times Y) \cong h_{*}(X) \otimes_{h_{*}} h_{*}(Y) .
$$

From now on we take all tensor products to be over the ground field $h_{*}$. We can therefore make the following definitions.

Definition 3. Fix $c \in \mathcal{C F}_{p, q}^{\mu}(g)$.

a. Define the push-forward map $\left(\rho_{i n}\right)_{!}: h_{*}(L M)^{\otimes p} \rightarrow h_{*+\chi\left(\Sigma_{c}\right) n}\left(M a p_{*}(c, M)\right)$ to be the composition

$$
\left(\rho_{i n}\right) !: h_{*}(L M)^{\otimes p} \cong h_{*}\left((L M)^{p}\right) \stackrel{\tau_{*}}{\longrightarrow} h_{*}\left(\operatorname{Map}_{*}(c, M)^{\nu\left(\Delta_{c}\right)}\right) \stackrel{t}{\stackrel{t}{\longrightarrow}} h_{*+\chi}\left(\Sigma_{c}\right) n\left(\operatorname{Map}_{*}(c, M)\right) .
$$

b. Define the operation $\mu_{c}: h_{*}(L M)^{\otimes p} \rightarrow h_{*}(L M)^{\otimes q}$ to be the composition,

$$
\mu_{c}: h_{*}(L M)^{\otimes p} \stackrel{\left(\rho_{i n}\right) !}{\longrightarrow} h_{*+\chi}\left(\Sigma_{c}\right) n\left(M a_{*}(c, M)\right) \stackrel{\left(\rho_{o u t}\right)_{*}}{\longrightarrow} h_{*+\chi\left(\Sigma_{c}\right) n}\left((L M)^{q}\right) .
$$

In order to use these operations to prove theorem 1 we will first need to verify the following. 
Theorem 4. The operations $\mu_{c}: h_{*}(L M)^{\otimes p} \rightarrow h_{*}(L M)^{\otimes q}$ do not depend on the choice of marked metric chord diagram $c \in \mathcal{C F}_{p, q}^{\mu}(g)$. In other words, they are invariants of the diffeomorphism type of the surface $\Sigma_{c}$.

Proof. We show that if $\gamma:[0,1] \rightarrow \mathcal{C F}_{p, q}^{\mu}(g)$ is a path of chord diagrams, then $\mu_{\gamma(0)}=\mu_{\gamma(1)}$. By the connectivity of $\mathcal{C F}_{p, q}^{\mu}(g)$ (theorem 2), this will prove the theorem. To do this we parameterize the construction of the operation. Namely, let

$$
\operatorname{Map}_{*}(\gamma, M)=\left\{(t, f): t \in[0,1], f \in \operatorname{Map}_{*}(\gamma(t), M)\right\} .
$$

Then there are restriction maps to the incoming and outgoing boundaries, $\rho_{\text {in }}: \operatorname{Map}_{*}(\gamma, M) \rightarrow$ $(L M)^{p}$, and $\rho_{\text {out }}: \operatorname{Map}_{*}(\gamma, M) \rightarrow(L M)^{q}$. Let $p: \operatorname{Map}_{*}(\gamma, M) \rightarrow[0,1]$ be the projection map. Then lemma 3 implies the following.

Lemma 5. The product $\rho_{\text {in }} \times p: \operatorname{Map}_{*}(\gamma, M) \hookrightarrow(L M)^{p} \times[0,1]$ is a codimension $-\chi\left(\Sigma_{c}\right) n$ embedding, and has an open neighborhood $\nu(\gamma)$ diffeomorphic to the total space of the vector bundle whose fiber over $(t, f) \in \operatorname{Map}_{*}(\gamma, M)$ is given by

$$
\nu(\gamma)_{(t, f)}=\bigoplus_{v \in \sigma(\gamma(t))} \bigoplus_{(\mu(v)-1)} T_{f(v)} M
$$

This allows us to define a Thom collapse map, $\tau:(L M)^{p} \times[0,1] \rightarrow\left(\operatorname{Map}_{*}(\gamma, M)\right)^{\nu(\gamma)}$ which defines a homotopy between the collapse maps $\tau_{0}:(L M)^{p} \rightarrow \operatorname{Map}_{*}(\gamma(0), M)^{\nu(\gamma(0))} \hookrightarrow \operatorname{Map}_{*}(\gamma, M)^{\nu(\gamma)}$ and $\tau_{1}:(L M)^{p} \rightarrow \operatorname{Map}_{*}(\gamma(1), M)^{\nu(\gamma(1))} \hookrightarrow \operatorname{Map}_{*}(\gamma, M)^{\nu(\gamma)}$.

One can then define the push-forward map,

$$
\left(\rho_{i n}\right)_{!}: h_{*}\left((L M)^{p} \times[0,1]\right) \stackrel{\tau_{*}}{\longrightarrow} h_{*}\left(\left(\operatorname{Map}_{*}(\gamma, M)\right)^{\nu(\gamma)}\right) \stackrel{t}{\cong} h_{*+\chi \cdot n}\left(\left(\operatorname{Map}_{*}(\gamma, M)\right)\right.
$$

and then an operation

$$
\mu_{\gamma}=\left(\rho_{\text {out }}\right)_{*} \circ\left(\rho_{\text {in }}\right) !: h_{*}\left((L M)^{p} \times[0,1]\right) \rightarrow h_{*+\chi \cdot n}\left(\left(\operatorname{Map}_{*}(\gamma, M)\right) \rightarrow h_{*+\chi \cdot n}\left((L M)^{q}\right) .\right.
$$

The restriction of this operation to $h_{*}\left((L M)^{p} \times\{0\}\right) \hookrightarrow h_{*}\left((L M)^{p} \times[0,1]\right)$ is, by definition, $\mu_{\gamma(0)}$, and the restriction to $h_{*}\left((L M)^{p} \times\{1\}\right) \hookrightarrow h_{*}\left((L M)^{p} \times[0,1]\right)$ is $\mu_{\gamma(1)}$. This proves that these two operations are equal.

Now that we have theorem 4 we can introduce the notation $\mu_{p, q}(g)$ to stand for $\mu_{c}: h_{*}(L M)^{\otimes p} \rightarrow$ $h_{*}(L M)^{\otimes q}$ for any Sullivan chord diagram $c \in \mathcal{C F}_{p, q}^{\mu}(g) . \mu_{p, q}(g)$ is an operation that lowers the total degree by $(2 g-2+p+q) n$.

In order to complete the proof of theorem [1] by the results of [9, [1, it suffices to show that these operations respect the gluing of surfaces. That is, it suffices to prove the following result.

Theorem 6. $\mu_{q, r}\left(g_{2}\right) \circ \mu_{p, q}\left(g_{1}\right)=\mu_{p, r}\left(g_{1}+g_{2}+q-1\right): h_{*}(L M)^{\otimes p} \rightarrow h_{*}(L M)^{\otimes q} \rightarrow h_{*}(L M)^{\otimes r}$. 


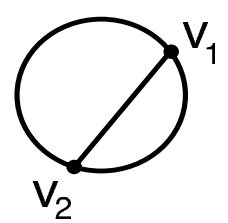

(a) $c_{1}$

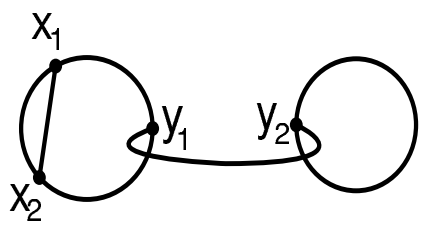

(b) $c_{2}$

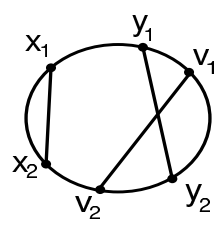

(c) $c_{1} \# c_{2}$

Figure 13: Gluing $c_{1}$ and $c_{2}$

Proof. Let $c_{1} \in \mathcal{C F}_{p, q}^{\mu}\left(g_{1}\right)$ and $c_{2} \in \mathcal{C F}_{q, r}^{\mu}\left(g_{2}\right)$. Notice that we can glue $c_{1}$ to $c_{2}$ to obtain a Sullivan chord diagram in $c_{1} \# c_{2} \in \mathcal{C} \mathcal{F}_{p, r}^{\mu}\left(g_{1}+g_{2}+q-1\right)$ in the following way.

Identify the outgoing boundary circles of $c_{1}$ with the incoming boundary circles of $c_{2}$ using the parameterizations, and input the vertices and ghost edges of $c_{2}$ into the diagram $c_{1}$ using these identifications. Figure 13 gives an example of this gluing procedure with $c_{1} \in \mathcal{C F}_{1,2}^{\mu}(0), c_{2} \in \mathcal{C F}_{2,2}^{\mu}(0)$, and $c_{1} \# c_{2} \in \mathcal{C F}_{1,2}^{\mu}(1)$. For clarity the vertices have been labeled in these diagrams, both before and after gluing.

Note. We are not claiming that this gluing procedure is continuous, or even well-defined. The ambiguity in definition occurs if, when one identifies the outgoing boundary circle of $c_{1}$ with an incoming boundary circle of $c_{2}$, a circular vertex $x$ of $c_{2}$ coincides with with a circular vertex $v$ of $c_{1}$ that lies on a ghost edge in the boundary cycle. Then there is an ambiguity over whether to place $x$ at $v$ or at the other vertex of the ghost edge. However for our purposes, we can make any such choice, since the operations that two such glued surfaces define are equal, by theorem 4

Notice that the parameterizations give us maps of the collapsed fat graphs,

$$
\phi_{1}: S\left(c_{1}\right) \rightarrow S\left(c_{1} \# c_{2}\right) \quad \text { and } \quad \phi_{2}: S\left(c_{2}\right) \rightarrow S\left(c_{1} \# c_{2}\right) .
$$

These induce a diagram of mapping spaces,

$$
\operatorname{Map}_{*}\left(c_{2}, M\right) \stackrel{\bar{\phi}_{2}}{\longleftarrow} \operatorname{Map}_{*}\left(c_{1} \# c_{2}, M\right) \stackrel{\bar{\phi}_{1}}{\longrightarrow} \operatorname{Map}_{*}\left(c_{1}, M\right)
$$

The next two lemmas follow from a verification of the definitions of the mapping spaces and the maps $\phi_{i}$.

Lemma 7. $\bar{\phi}_{1}: \operatorname{Map}_{*}\left(c_{1} \# c_{2}, M\right) \rightarrow \operatorname{Map}_{*}\left(c_{1}, M\right)$ is an embedding, whose image has a neighborhood diffeomorphic to the total space of the bundle $\bar{\phi}_{2}^{*}\left(\nu\left(c_{2}\right)\right)$, where $\nu\left(c_{2}\right) \rightarrow \operatorname{Map}_{*}\left(c_{2}, M\right)$ is the normal bundle of $\rho_{\text {in }}: \operatorname{Map}_{*}\left(c_{2}, M\right) \hookrightarrow(L M)^{p}$ described in lemma 3

This allows the definition of a Thom collapse map $\tau_{\phi_{1}}: \operatorname{Map}_{*}\left(c_{1}, M\right) \rightarrow \operatorname{Map}_{*}\left(c_{1} \# c_{2}, M\right)^{\bar{\phi}_{2}^{*}\left(\nu\left(c_{2}\right)\right)}$ and therefore a push-forward map in homology, $\left(\bar{\phi}_{1}\right) !: h_{*}\left(\operatorname{Map}_{*}\left(c_{1}, M\right)\right) \rightarrow h_{*+\chi\left(c_{2}\right) n}\left(\operatorname{Map}_{*}\left(c_{1} \# c_{2}, M\right)\right)$. 
Lemma 8. The following diagram commutes:

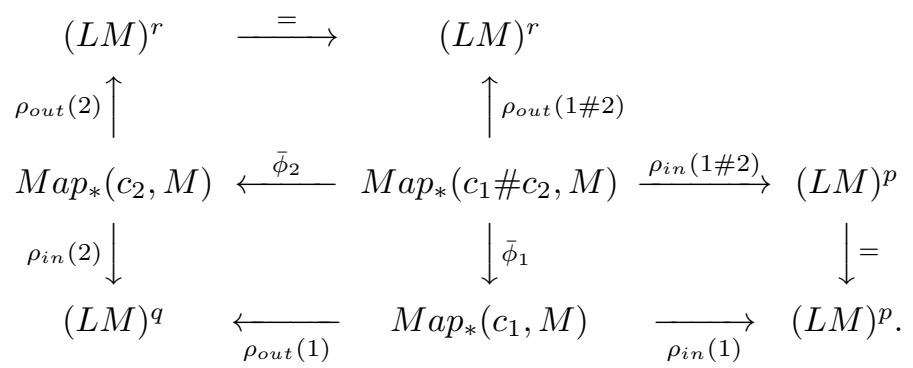

The indexing of the restriction maps corresponds to the indexing of the chord diagrams in the obvious way.

By the naturality of the Thom collapse map, and therefore the homological pushout construction, we therefore have the following corollary.

Corollary 9. 1. $\left(\rho_{\text {in }}(1 \# 2)\right) !=\left(\bar{\phi}_{1}\right) ! \circ\left(\rho_{\text {in }}(1)\right) !: h_{*}\left((L M)^{p}\right) \rightarrow h_{*+\chi}\left(c_{1} \# c_{2}\right) \cdot n\left(M_{a p}\left(c_{1} \# c_{2}, M\right)\right)$

2. $\left(\bar{\phi}_{2}\right)_{*} \circ\left(\bar{\phi}_{1}\right) !=\left(\rho_{\text {in }}(2)\right) ! \circ\left(\rho_{\text {out }}(1)\right)_{*}: h_{*}\left(\operatorname{Map}\left(c_{1}, M\right)\right) \rightarrow h_{*+\chi}\left(c_{2}\right) \cdot n\left(\operatorname{Map}_{*}\left(c_{2}, M\right)\right)$

3. $\left(\rho_{\text {out }}(1 \# 2)\right)_{*}=\left(\rho_{\text {out }}(2)\right)_{*} \circ\left(\bar{\phi}_{2}\right)_{*}: h_{*}\left(\operatorname{Map}_{*}\left(c_{1} \# c_{2}, M\right)\right) \rightarrow h_{*}\left((L M)^{r}\right)$.

We may now complete the proof of theorem [6 We have

$$
\begin{aligned}
\mu_{p, r}\left(g_{1}+g_{2}+q-1\right)=\mu_{c_{1} \# c_{2}} & =\left(\rho_{\text {out }}(1 \# 2)\right)_{*} \circ\left(\rho_{\text {in }}(1 \# 2)\right) ! \\
& =\left(\rho_{\text {out }}(1 \# 2)\right)_{*} \circ\left(\bar{\phi}_{1}\right) ! \circ\left(\rho_{\text {in }}(1)\right)_{!} \\
& =\left(\rho_{\text {out }}(2)\right)_{*} \circ\left(\bar{\phi}_{2}\right)_{*} \circ\left(\bar{\phi}_{1}\right) ! \circ\left(\rho_{\text {in }}(1)\right) ! \\
& =\left(\rho_{\text {out }}(2)\right)_{*} \circ\left(\rho_{\text {in }}(2)\right) ! \circ\left(\rho_{\text {out }}(1)\right)_{*} \circ\left(\rho_{\text {in }}(1)\right) ! \\
& =\mu_{c_{2}} \circ \mu_{c_{1}} \\
& =\mu_{q, r}\left(g_{2}\right) \circ \mu_{p, q}\left(g_{1}\right) .
\end{aligned}
$$

As observed in 1, a Frobenius algebra without counit is the same thing as a positive boundary topological quantum field theory. We have now verified that the string topology operations define such a theory for any generalized cohomology theory $h^{*}$ satisfying the conditions described above. Recall that it was observed in [3] [5], that the unit in the algebra structure of $h_{*}(M)$ is the fundamental class, $[M] \in h_{n}(M) \hookrightarrow h_{n}(L M)$, where the second map is induced by the inclusion of the manifold in the loop space as the constant loops, $\iota: M \hookrightarrow L M$. Thus $h_{*}(L M)$ is a unital Frobenius algebra without a counit. This proves theorem 1 


\section{Capping off boundary components: issues surrounding the unit and counit}

The unit in the Frobenius algebra stucture can be constructed in the same way as the other string topology operations as follows.

Consider the disk $D^{2}$ as a surface with zero incoming boundary component and one outgoing boundary component. A graph $c_{D}$ that represents $D^{2}$ can be taken to be a point (i.e a single vertex). Formally, the restriction to the zero incoming boundary components is the map

$$
\rho_{\text {in }}: M=\operatorname{Map}_{*}\left(c_{D}, M\right) \rightarrow M a p(\emptyset, M)=\text { point }
$$

The push-forward map in this setting

$$
\left(\rho_{\text {in }}\right) !: h_{*}(\text { point }) \rightarrow h_{n}(M)
$$

is the $h_{*}$-module map defined by sending the generator to $[M] \in h_{n}(M)$. The restriction to the outgoing boundary component is the map

$$
\rho_{\text {out }}: M=\operatorname{Map}_{*}\left(c_{D}, M\right) \rightarrow L M
$$

which is given by $\iota: M \hookrightarrow L M$. Thus the unit is given by the $h_{*}$ module homomorphism

$$
\mu_{D^{2}}=\left(\rho_{\text {out }}\right)_{*} \circ\left(\rho_{\text {in }}\right) !=\iota_{*} \circ\left(\rho_{\text {in }}\right) !: h_{*} \rightarrow h_{n}(M) \rightarrow h_{n}(L M)
$$

which sends the generator to the fundamental class.

The issue of the existence (or nonexistence) of a counit in the Frobenius algebra structure given by theorem 1 is formally the same (or dual) to the existence of a unit, but is geometrically much more difficult and subtle. Namely, for this operation one must consider $D^{2}$ as a surface with one incoming boundary, and zero outgoing boundary components. In this setting the roles of the restriction maps $\rho_{\text {in }}$ and $\rho_{\text {out }}$ are reversed, and one obtains the diagram

$$
\operatorname{Map}(\emptyset, M) \stackrel{\rho_{\text {out }}}{\longleftarrow} \operatorname{Map}_{*}\left(c_{D}, M\right) \stackrel{\rho_{\text {in }}}{\longrightarrow} L M
$$

or, equivalently,

$$
\text { point } \stackrel{\epsilon}{\longleftarrow} M \stackrel{\iota}{\longrightarrow} L M
$$

where $\epsilon: M \rightarrow$ point is the constant map.

Now notice that in this case, unlike when any of the other fat graphs were considered, the embedding of $\operatorname{Map}_{*}\left(c_{D}, M\right) \hookrightarrow L M$ (i.e $\left.\iota: M \hookrightarrow L M\right)$ is of infinite codimension. Therefore to find a theory $h_{*}$ that supports a counit in the Frobenius algebra structure of $h_{*}(L M)$, one needs to be able to define a push-forward map for this infinite codimensional embedding. Now in their work on genera of loop spaces, 2], Ando and Morava argued that if one has a theory where this push-forward 
map exists, one would need that the Euler class of the normal bundle $e(\nu(\iota)) \in h^{*}(M)$ is invertible. So let us now consider this normal bundle.

The embedding of $M$ as the constant loops in $L M$ is $S^{1}$-equivariant where $S^{1}$ acts triviallly on $M$. When $M$ is a simply connected almost complex manifold, the normal bundle has the following description (see [2], for example).

Lemma 10. The normal bundle $\nu(\iota) \rightarrow M$ of the embedding $\iota: M \hookrightarrow L M$ is equivariantly isomorphic to the direct sum,

$$
\nu(\iota) \cong \bigoplus_{k \neq 0} T M \otimes_{\mathbb{C}} \mathbb{C}(k)
$$

where $\mathbb{C}(k)$ is the one dimensional representation of $S^{1}$ of weight $k$.

This says that the Euler class of the normal bundle will have the formal description

$$
e(\nu(\iota))=\prod_{k \neq 0} e(T M \otimes \mathbb{C}(k)) .
$$

Thus a theory $h_{*}(L M)$ that supports a counit in a Frobenius algebra structure should have the following properties.

1. $h_{*}$ should be an $S^{1}$ - equivariant theory in order to take advantage of the different equivariant structures of the summands $T M \otimes \mathbb{C}(k)$.

2. $h_{*}$ should be a "pro-object" - an inverse system of homology groups, so that it can accommodate this infinite product.

\section{The polarized loop space and its Atiyah dual}

Motivated by these homological requirements, in this section we show that the loop space of an almost complex manifold has a natural equivariant pro-object (a "prospectrum") associated to it. The ideas for the constructions in this section stem from conversations with Graeme Segal. Throughout this section we assume that $M$ is a simply connected, oriented, closed $n$-manifold.

Let $-T M$ be the virtual bundle ( $K$-theory class) given by the opposite of the tangent bundle. Let $M^{-T M}$ be its Thom spectrum. We refer to $M^{-T M}$ as the "Atiyah dual" of $M_{+}$because of Atiyah's well known theorem stating that $M^{-T M}$ is equivalent to the Spanier Whitehead dual of $M_{+}$. (Here $M_{+}$is $M$ together with a disjoint basepoint.) This gives $M^{-T M}$ the structure of a ring spectrum, whose multiplication $m: M^{-T M} \wedge M^{-T M} \rightarrow M^{-T M}$ is dual to the diagonal $\Delta: M \rightarrow M \times M$. When one applies homology and the Thom isomorphism, this multiplication realizes the intersection product $(\cap)$, meaning that the following diagram commutes.

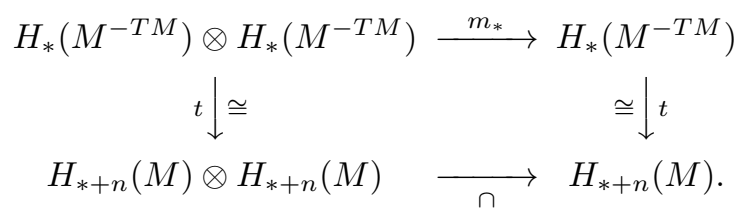


This was used in [5] to show that the Thom spectrum $L M^{-T M}$ of the pullback of $-T M$ via the evaluation map $e_{1}: L M \rightarrow M$, is a ring spectrum whose multiplication realizes the Chas-Sullivan loop homology product, $\mu_{*}: H_{*}(L M) \otimes H_{*}(L M) \rightarrow H_{*-n}(L M)$. It was also used in [7] to define a spectral sequence for calculating the loop homology algebra.

It is therefore natural to expect that an appropriate pro-object that carries the string topology operations, including a counit, (i.e a 2 dimensional TQFT, or Frobenius algebra structure), would be a prospectrum model for the Atiyah dual of the loop space, $L M^{-T L M}$. Notice that since $T L M$ is an infinite dimensional bundle, it does not represent a $K$-theory class, so one cannot simply define a virtual bundle, "-TLM". Therefore $L M^{-T L M}$ cannot simply be a spectrum but rather we must define a pro-spectrum, or an inverse limit of spectra, to model this Atiyah dual.

In studying homotopy theoretic aspects of symplectic Floer homology, the first author, Jones and Segal used pro-spectra associated to certain infinite dimensional bundles [6]. The construction was the following. If $E \rightarrow X$ is an infinite dimensional bundle with a filtration by finite dimensional subbundles,

$$
\cdots \hookrightarrow E_{i} \hookrightarrow E_{i+1} \hookrightarrow \cdots E
$$

such that $\bigcup_{i} E_{i}$ is a dense subbundle of $E$, then one can define the prospectrum $X^{-E}$ to be the inverse system,

$$
\cdots \leftarrow X^{-E_{i-1}} \stackrel{u_{i}}{\longleftarrow} X^{-E_{i}} \stackrel{u_{i+1}}{\longleftarrow} X^{-E_{i+1}} \leftarrow \cdots
$$

where $u_{j}: X^{-E_{j}} \rightarrow X^{-E_{j-1}}$ is the map defined as follows. Let $e_{j}: E_{j-1} \hookrightarrow E_{j}$ be the inclusion. Assume for simplicity that $E_{j}$ is embedded in a large dimensional trivial bundle, and let $E_{j}^{\perp}$ and $E_{j-1}^{\perp}$ be the corresponding orthogonal complements. One then has an induced inclusion of complements, $e_{j}^{\perp}: E_{j}^{\perp} \rightarrow E_{j-1}^{\perp}$. The induced map of Thom spaces then defines a map of Thom spectra, $u_{j}:$ $X^{-E_{j}} \rightarrow X^{-E_{j-1}}$. A standard homotopy theoretic technique allows one to define this map of Thom spectra even if $E_{j}$ is not embeddable in a trivial bundle, by restricting $E_{j}$ to finite subcomplexes of $X$ where it is.

Under the assumption that $M$ is an almost complex manifold of dimension $n=2 m$, then we are dealing with an infinite dimensional vector bundle (TLM) whose structure group is the loop group $L U(m)$. In $[8$, Cohen and Stacey studied obstructions to finding an appropriate filtration of an infinite dimensional $L U(m)$ bundle. In particular for $T L M \rightarrow L M$ it was proved that if such a filtration (called a "Fourier decomposition" in [8]) exists, then the holonomy of any unitary connection on $T M, h: \Omega M \rightarrow U(m)$ is null homotopic. This "homotopy flat" condition is far too restrictive for our purposes, but we can get around this problem by taking into account the canonical polarization of the tangent bundle $T L M$ of an almost complex manifold.

Recall that a polarization of a Hilbert space $E$ is an equivalence class of decomposition, $E=$ $E_{+} \oplus E_{-}$, where two such decompositions $E_{+} \oplus E_{-}=E_{+}^{\prime} \oplus E_{-}^{\prime}$ are equivalent if the composition $E_{+} \hookrightarrow E \rightarrow E_{+}^{\prime}$ is Fredholm, and $E_{+} \hookrightarrow E \rightarrow E_{-}^{\prime}$ is compact. (See 14 for details.) Roughly speaking a polarization is a splitting of $E$ which is well defined up to finite dimensional ambiguity. 
Such polarizations arise from unbounded self adjoint operators, $D: E \rightarrow E$. This splits $E$ according to the positive and negative eigenspaces of $D$. The finite dimensional ambiguity appears in the choice of how to split the zero eigenspace among the positive and negative summands. The restricted general linear group of a polarized space $G L_{r e s}(E)$ consists of all elements of $G L(E)$ that preserve the polarization.

A polarized vector bundle $\zeta \rightarrow X$ is one where every fiber is polarized, and the structure group reduces to the restricted general linear group. If $M^{2 m}$ is an almost complex manifold, and $\gamma \in L M$, then the tangent space $T_{\gamma} L M$ is the space of $L^{2}$ vector fields of $M$ along $\gamma$, and the operator

$$
j \frac{d}{d \theta}: T_{\gamma} L M \rightarrow T_{\gamma} L M
$$

is a self adjoint Fredholm operator. Here $\frac{d}{d \theta}$ is the covariant derivative, and $j$ is the almost complex structure. The spectral decomposition of $j \frac{d}{d \theta}$ polarizes the bundle $T L M$. The structure group in this case is $G L_{r e s}\left(L^{2}\left(S^{1}, \mathbb{C}^{m}\right)\right)$, where the loop space $L^{2}\left(S^{1}, \mathbb{C}^{m}\right)$ is polarized according to the Fourier decompostion. That is, we write

$$
L^{2}\left(S^{1}, \mathbb{C}^{n}\right)=H_{+} \oplus H_{-}
$$

where $H_{+}=\operatorname{Hol}\left(D^{2}, \mathbb{C}^{n}\right)$ is the space of holomorphic maps of the disk, and $H_{-}$is the orthogonal complement.

For a polarized space $E$, recall from [14] that the restricted Grassmannian $G r_{r e s}(E)$ consists of closed subspaces $W \subset E$ such that the projections $W \hookrightarrow E \rightarrow E_{+}$is Fredholm, and $W \hookrightarrow E \rightarrow E_{-}$ is Hilbert-Schmidt. (Notice that this definition does not depend on the choice of decomposition in the polarization class of $E$.) In the case under consideration, the tangent space $T_{\gamma} L M$, is a $L \mathbb{C}$ module, and therefore a module over the Laurent polynomial ring, $\mathbb{C}\left[z, z^{-1}\right]$. Define $G r_{r e s}^{0}\left(T_{\gamma} L M\right) \subset$ $G r_{r e s}\left(T_{\gamma} L M\right)$ to be the subspace

$$
G r_{r e s}^{0}\left(T_{\gamma} L M\right)=\left\{W \in G r_{r e s}\left(T_{\gamma} L M\right): z W \subset W\right\}
$$

For $M^{2 m}$ a simply connected almost complex manifold, we can then define the polarized loop space $L M_{ \pm}$to be the space

$$
L M_{ \pm}=\left\{(\gamma, W): \gamma \in L M, W \in G r_{r e s}^{0}\left(T_{\gamma} L M\right)\right\} .
$$

We now consider the $S^{1}$-equivariance properties of $L M_{ \pm}$. To do this we recall some notation from [14, chapters 7 and 8. First, define $\mathbb{S}$ to the set of all subsets $S \subset \mathbb{Z}$ whose symmetric difference with the nonnegative integers, $\mathbb{Z}^{+}$is finite. That is,

$$
S \Delta \mathbb{Z}^{+}=\left\{S-\mathbb{Z}^{+}\right\} \cup\left\{\mathbb{Z}^{+}-S\right\}
$$

is a finite set. The virtual cardinal of such a set $S, v \cdot c(S)$ is defined to be

$$
v . c(S)=\#\left\{S-\mathbb{Z}^{+}\right\}-\#\left\{\mathbb{Z}^{+}-S\right\} .
$$


A set $S$ with virtual cardinal v.c. $(S)=k$ will be of type $n$, if for every $s \in S$,

$$
s+n \in S .
$$

Consider the orthonormal basis of the Hilbert space $H=L^{2}\left(S^{1}, \mathbb{C}\right)$ given by $\left\{z^{k}\right\}_{k \in \mathbb{Z}}$. For $S \subset \mathbb{S}$, there is a distinguished point $\left.H_{S} \in G r_{r e s}(H)\right)$ defined to be the closed subspace spanned by $z^{k}$ for $k \in S$.

Theorem 11. The natural projection $p: L M_{ \pm} \rightarrow L\left(M^{2 m}\right)$ is an $S^{1}$ equivariant fiber bundle with fiber diffeomorphic to the based loop space, $\Omega U(m)$. The $S^{1}$-fixed points of $L M_{ \pm}$are homeomorphic to $M \times \mathbb{S}_{m}$, where $\mathbb{S}_{m} \subset \mathbb{S}$ consists of all $S \in \mathbb{S}$ of type $m$.

Proof. Let $\gamma \in L M$. The tangent space, $T_{\gamma} L M=\Gamma_{S^{1}}\left(\gamma^{*}(T M)\right)$, is the space of $L^{2}$ sections of the pullback of the tangent bundle over the circle. The $S^{1}$ - action on $L M$ differentiates to make the tangent bundle $T L M$ an $S^{1}$-equivariant bundle. If $\sigma \in T_{\gamma} L M$, and $t \in S^{1}$ then $t \sigma \in T_{t \gamma} L M$ is defined by $t \sigma(s)=\sigma(t+s)$. Since this action preserves the polarization, it induces an action

$$
\begin{aligned}
& S^{1} \times L M_{ \pm} \rightarrow L M_{ \pm} \\
& t \times(\gamma, W) \rightarrow(t \gamma, t W)
\end{aligned}
$$

where $t W=\left\{t \sigma \in T_{t \gamma} L M: \sigma \in W\right\}$. The fact that the projection map $p: L M_{ \pm} \rightarrow L M$ is an $S^{1}$-equivariant bundle is clear. The fiber of this bundle can be identified with $G r_{r e s}^{0}\left(L^{2}\left(S^{1}, \mathbb{C}^{m}\right)\right)$, which was proved in [14] to be diffeomorphic to $\Omega U(m)$.

We now consider the fixed points of the $S^{1}$-action on $L M_{ \pm}$. Take $(\gamma, W) \in L M_{ \pm}$to be one of them. Notice that $\gamma$ must be a constant loop at some point, $x \in M$. But the tangent space at such a constant loop is given by $T_{x} L M=L^{2}\left(S^{1}, \mathbb{C}\right) \otimes_{\mathbb{C}} T_{x} M$, with the circle acting on the left factor. So we want to compute the fixed points of the $S^{1}$ action on $\operatorname{Gr}_{\text {res }}^{0}\left(L^{2}\left(S^{1}, \mathbb{C}\right) \otimes_{\mathbb{C}} T_{x} M\right)$, or equivalently, on $G r_{r e s}^{0}\left(L^{2}\left(S^{1}, \mathbb{C}^{m}\right)\right)$. For ease of notation, let $H=L^{2}\left(S^{1}, \mathbb{C}\right)$, and $H^{(m)}=L^{2}\left(S^{1}, \mathbb{C}^{m}\right)$. It was shown in [14 that the fixed points of the $S^{1}$-action on $G r_{r e s}(H)$ are the points $H_{S}$ with $S \in \mathbb{S}$. Now the Hilbert space $H^{m}$ can be identified with $H=L^{2}\left(S^{1}, \mathbb{C}\right)$ by corresponding their standard orthonormal bases using a lexicographical ordering. More precisely, let $\left\{e_{i}, i=1, \cdots, m\right\}$ be the standard basis for $\mathbb{C}^{m}$. Then make $e_{i} z^{k} \in H^{(m)}$ correspond to $z^{m k+i-1} \in H$. Under this identification, multiplication by $z$ in $H^{(m)}=L^{2}\left(S^{1}, \mathbb{C}^{m}\right)$ corresponds to multiplication by $z^{m}$ in $H=L^{2}\left(S^{1}, \mathbb{C}\right)$. So the fixed points of the action of $S^{1}$ on $G r_{r e s}^{0}\left(H^{(m)}\right)$ correspond to the fixed points of the action of $S^{1}$ on $G r_{r e s}(H)$ that are invariant under multiplication by $z^{m}$. These are precisely those $H_{S}$ with $S \in \mathbb{S}$ such that $s+m \in S$ for every $s \in S$. That is the fixed points are those $H_{S}$ with $S \in \mathbb{S}_{m}$.

This proves that the projection map $p: L M_{ \pm} \rightarrow L M$ restricts to the fixed point sets to define a covering of the constant loops $M \subset L M$ whose fiber is the discrete set $\mathbb{S}_{m}$. Since $M$ is simply connected the theorem follows. 
Remark. Since the $S^{1}$ - fixed points of $L M_{ \pm}$are the $S^{1}$-fixed points of $L M$ times a finite set, one can view the above theorem as saying that the equivariant homotopy type of $L M_{ \pm}$is directly computable in terms of the equivariant homotopy type of $L M$.

By this theorem, the pullback of the tangent bundle, $p^{*} T L M \rightarrow L M_{ \pm}$is an $S^{1}$ equivariant bundle. Our final result implies that even though one cannot generally find a prospectrum modeling the Atiyah dual $L M^{-T L M}$, one can find a pro-spectrum model of the "polarized Atiyah dual", $L M_{ \pm}^{-T L M}$.

The following theorem says that one can build up the bundle $p^{*}(T L M) \rightarrow L M_{ \pm}$by finite dimensional subbundles.

Theorem 12. There is a doubly graded collection of finite dimensional, $S^{1}$-equivariant subbundles of $p^{*}(T L M) \rightarrow L M_{ \pm}$,

$$
E_{i, j} \rightarrow L M_{ \pm}, \quad i<j
$$

satisfying the following properties:

1. There are inclusions of subbundles

$$
E_{i, j} \hookrightarrow E_{i-1, j} \quad \text { and } \quad E_{i, j} \hookrightarrow E_{i, j+1}
$$

such that $\bigcup_{i, j} E_{i, j}$ is a dense subbundle of $p^{*}(T L M)$.

2. The subquotients,

$$
E_{i-1, j} / E_{i, j} \text { and } E_{i, j+1} / E_{i, j}
$$

are $m$ dimensional $S^{1}$-equivariant complex vector bundles that are nonequivariantly isomorphic to the pullback of the tangent bundle $\tilde{p}^{*} T M$, where $\tilde{p}: L M_{ \pm} \rightarrow M$ is the composition of $p: L M_{ \pm} \rightarrow L M$ with the map $e_{1}: L M \rightarrow M$ that evaluates a loop at the basepoint $1 \in S^{1}$.

Remark. Such a filtration is a "Fourier decomposition" of the loop bundle $p^{*}(T L M) \rightarrow L M_{ \pm}$ as defined in 8 ]

Proof. We first define certain infinite dimensional subbundles $E_{i} \subset p^{*}(T L M) \rightarrow L M_{ \pm}$. Define the fiber over $(\gamma, W) \in L M_{ \pm}$to be

$$
\left(E_{i}\right)_{(\gamma, W)}=z^{-i} W \subset T_{\gamma}(L M) .
$$

We note that $E_{i}$ is an equivariant subbundle, with the property that $z E_{i} \subset E_{i}$. Furthermore there is a filtration of subbundles

$$
\cdots \hookrightarrow E_{i} \hookrightarrow E_{i+1} \hookrightarrow \cdots p^{*}(T L M)
$$

with $\bigcup_{i} E_{i}$ a dense subbundle of $p^{*}(T L M)$. Notice that for $j>i$, the subquotient $E_{j} / E_{i}$ has fiber at $(\gamma, W)$ given by $z^{-j} W \cap\left(z^{-i} W\right)^{\perp}$ where $\left(z^{-i} W\right)^{\perp} \subset T_{\gamma} L M$ is the orthogonal complement of $z^{-i} W$. For $j-i=1$, an easy argument (done in [8]) gives that the composition

$$
z^{-j} W \cap\left(z^{-(j-1)} W\right)^{\perp} \hookrightarrow T_{\gamma} L M \stackrel{e_{1}}{\longrightarrow} T_{\gamma(1)}(M)
$$


is an isomorphism. For $i<j$ we define the bundle $E_{i, j} \rightarrow L M_{ \pm}$to be the quotient $E_{j} / E_{i}$. It is the vector bundle whose fiber over $(\gamma, W)$ is $z^{-j} W \cap\left(z^{-i} W\right)^{\perp}$. By (12), the subquotient the bundle $E_{j-1 . j}$ is (nonequivariantly) isomorphic to the pullback of the tangent bundle $T M \rightarrow M$ under the composition $L M_{ \pm} \stackrel{p}{\longrightarrow} L M \stackrel{e_{1}}{\longrightarrow} M$. In general the bundle $E_{i, j}$ is nonequivariantly isomorphic to the Whitney sum of $j-i$ copies of $\tilde{p}^{*}(T M)$.

Now since $z^{-j} W \cap\left(z^{-i} W\right)^{\perp}$ is a subspace of both $z^{-(j+1)} W \cap\left(z^{-i} W\right)^{\perp}$ and of $z^{-j} W \cap\left(z^{-(i-1)} W\right)^{\perp}$, we have inclusions $E_{i, j} \hookrightarrow E_{i, j+1}$ and $E_{i, j} \hookrightarrow E_{i-1, j}$. Clearly $\bigcup_{i, j} E_{i, j}$ is a dense subbundle of $p^{*}(T L M)$. The theorem follows.

Since the bundles $E_{i, j} \rightarrow L M_{ \pm}$are finite dimensional $S^{1}$-equivariant bundles, we can construct the Thom spectrum of the $S^{1}$-equivariant virtual bundle, $-E_{i, j}$, which we denote by $\left(L M_{ \pm}\right)^{-E_{i, j}}$. Notice the inclusions of bundles $E_{i, j} \hookrightarrow E_{i, j+1}$ and $E_{i, j} \hookrightarrow E_{i-1, j}$ induce maps of virtual bundles, $\tau_{i, j}:-E_{i, j+1} \rightarrow-E_{i, j}$ and $\sigma_{i, j}:-E_{i-1, j} \rightarrow-E_{i, j}$, which yields an inverse system of $S^{1}$-equivariant spectra,

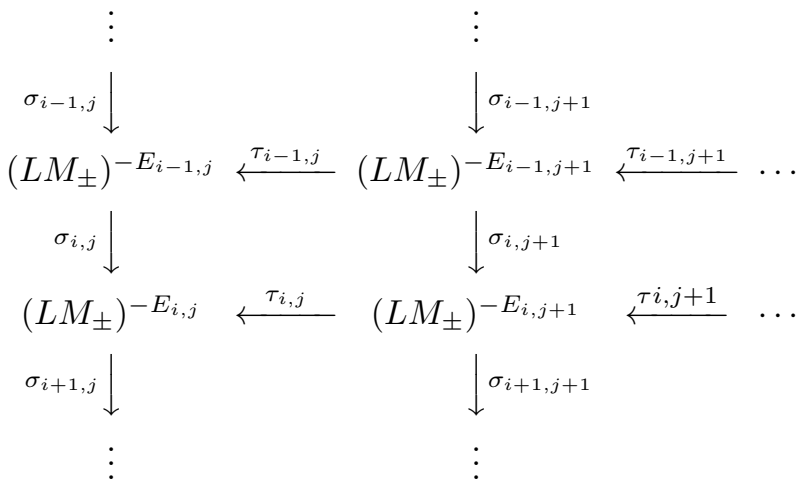

This system defines a pro-object in the category of $S^{1}$-equivariant spectra that we call the polarized Atiyah dual, $L M_{ \pm}^{-T L M}$. If one applies an equivariant homology theory to this prospectrum, one gets a pro-object in the category of graded abelian groups. Notice that in cohomology, the structure maps $\tau_{i, j}$ and $\sigma_{i, j}$ will induce multiplication by the equivariant Euler classes of the orthogonal complement bundles of these inclusions. As seen above, these orthogonal complement bundles are nonequivariantly isomorphic to the pull back of the tangent bundle, $T M$. However they have different equivariant structures. In future work we will study those equivariant cohomology theories for which these Euler classes are units, with the goal being to prove that such theories, when applied to this prospectrum, support the string topology operations including one that corresponds to a disk viewed as a cobordism from a circle to the empty set. By gluing, this will allow the construction of string topology operations for closed surfaces as well as surfaces with a positive number of outgoing boundary components. 


\section{References}

[1] L. Abrams, Two dimensional topological quantum field theories and Frobenius algebras, J. Knot theory ramifications 5 (1996), 569-587.

[2] M. Ando and J. Morava, A renormalized Riemann-Roch formula and the Thom isomorphism for the free loop space, Topology, geometry, and algebra: interactions and new directions (Stanford, CA, 1999), 11-36, Contemp. Math., 279, Amer. Math. Soc., Providence, RI, 2001.

[3] M. Chas and D. Sullivan, String Topology, to appear in Annals of Math., preprint: math.GT/9911159. (1999).

[4] M.Chas and D. Sullivan, Closed string operators in topology leading to Lie bialgebras and higher string algebra, preprint: math.GT/0212358 (2002).

[5] R.L. Cohen and J.D.S. Jones, A homotopy theoretic realization of string topology, Math. Annalen, published online: DOI 10.1007/s00208-002-0362-0 (2002). Preprint: math.GT0107187.

[6] R.L. Cohen, J.D.S. Jones, and G. Segal, Floer's infinite dimensional Morse theory and homotopy theory, Floer Memorial Volume, Birkhauser Verlag Prog. in Math. vol. 133 (1995), 287 - 325.

[7] R.L. Cohen, J.D.S. Jones, and J. Yan, The loop homology algebra of spheres and projective spaces, to appear in Proc. of conference on algebraic topology, Skye, Scotland, 2001. Preprint: math.AT/0210353

[8] R.L. Cohen and A. Stacey, Fourier decompositions of loop bundles, preprint: math/0210351, 2002

[9] R. Dijkgraaf, A geometric approach to two dimensional conformal field theory, PhD thesis, Univ. of Utrecht, (1989).

[10] V. Godin, Stanford University Ph.D thesis, in preparation.

[11] K. Igusa, Higher Franz-Reidemeister Torsion, AMS/IP Studies in Adv. Math., vol 31, International Press, 2002.

[12] K. Igusa, Combinatorial Miller-Morita-Mumford classes and Witten cycles, preprint: math.GT/0207042 (2002).

[13] R. Penner, The decorated Teichmuller space of punctured surfaces, Comm. Math. Phys. 113 (1987), 299-339.

[14] A. Pressley and G. Segal Loop Groups, Oxford Math. Monographs, Clarendon Press (1986).

[15] G. Segal Two dimensional conformal field theories and modular functors, in IXth Int'l. Congress on Math. Physics (1988) 22-37. 
[16] K. Strebel, Quadratic Differentials, Springer Verlag, Berlin, 1984.

[17] D. Sullivan, Closed string operators and Feynman graphs, in preparation.

[18] A. Voronov, Notes on universal algebra, to appear in proceedings of Stony Brook conf. on "Graphs and Patterns in Mathematics and Physics", June 2001. Preprint: math.QA/0111009. 